\title{
Comunidade de escarabeíneos (Coleoptera, Scarabaeidae) copro-necrófagos da região de Brejo Novo, Caruaru, Pernambuco, Brasil
}

\author{
Fernando Augusto Barbosa Silva ${ }^{1}$, Malva Isabel Medina Hernández², Sergio Ide ${ }^{3}$ Rita de Cássia de Moura ${ }^{1}$
}

${ }^{1}$ Departamento de Biologia, Instituto de Ciências Biológicas, Universidade de Pernambuco, Rua Arnóbio Marques 310, $50100-130$ Recife-PE, Brasil. fernandoabsilva@yahoo.com.br; rita_upe@yahoo.com.br

${ }^{2}$ Programa Regional de Pós-Graduação em Desenvolvimento e Meio Ambiente, Universidade Federal da Paraíba, Caixa Postal 5122, 58051-970 João Pessoa-PB, Brasil. malvamh@yahoo.com

${ }^{3}$ Centro de Pesquisa e Desenvolvimento de Sanidade Vegetal, Instituto Biológico, Av. Conselheiro Rodrigues Alves 1252, 04014-900 São Paulo-SP, Brasil.ide@biologico.sp.gov.br

\begin{abstract}
Copro-necrophagous scarab community (Coleoptera, Scarabaeidae) of the Brejo Novo region, Caruaru, Pernambuco, Brazil. This paper reports the results of the preliminary survey of copro-necrophagous dung beetles of Brejo Novo region, Caruaru, Pernambuco, as well as some aspects of the community structure such as seasonality, diversity, equitability, richness and abundance of species. Ten samplings with intervals of 30 days and duration of 48 hours, fortnightly were done between September 2003 to July 2004. Scarab beetles were collected with 24 pitfall traps baited with two types of baits, human excrements and rotting bovine meat. A total of 1,540 individuals belonging to six tribes, 12 genera and 28 species were collected. The species Canthon af. carbonarius, Canthon chalybaeus, Dichotomius nisus, D. semisquamosus, Digitonthophagus gazella and Eurysternus hirtellus are, apparently, adapted to the studied environment. Traps baited with human excrements attracted 826 individuals and those with rotting bovine meat attracted 714 specimens. Large number of species (15) considered "rare" occurred, being three of them "singletons", two "doubletons" and 10 with abundance between three and 10 individuals; 13 species were considered "common". Of the analyzed species, seven present generalist alimentary habits, four are strictly coprophagous and one is strictly necrophagous. Positive correlations were verified between the precipitation and the abundance of the individuals and the species richness. This preliminary survey of the dung beetles of the Brejo Novo region contributed to increase the number of species registered for Pernambuco and northeast region of Brazil.
\end{abstract}

KEYWORDS. Alimentary habit; biodiversity; ecology; scarabaeinae.

RESUMO. Comunidade de escarabeíneos (Coleoptera, Scarabaeidae) copro-necrófagos da região de Brejo Novo, Caruaru, Pernambuco, Brasil. Este estudo objetivou levantar preliminarmente os escarabeíneos copro-necrófagos da região de Brejo Novo, Caruaru, Pernambuco, e verificar aspectos da estrutura da comunidade como sazonalidade, diversidade, equitabilidade, riqueza e abundância das espécies. Foram realizadas 10 coletas, com intervalos de 30 dias e duração de 48 horas, de setembro de 2003 a julho de 2004. Utilizaram-se 24 armadilhas de queda, com dois tipos de isca, fezes humanas e carne bovina em estado de putrefação. Foram coletados 1.540 indivíduos pertencentes a seis tribos, 12 gêneros e 28 espécies. As espécies Canthon af. carbonarius, Canthon chalybaeus, Dichotomius nisus, D. semisquamosus, Digitonthophagus gazella e Eurysternus hirtellus aparentemente são as mais adaptadas ao ambiente estudado. Foram coletados 826 indivíduos em armadilhas iscadas com fezes humanas e 714 em carne bovina apodrecida. Ocorreu um número maior de espécies consideradas "raras" (15), sendo três destas "singletons", duas "doubletons" e 10 com abundância entre três e 10 indivíduos, 13 espécies foram consideradas "comuns". Das espécies analisadas, sete apresentaram hábitos alimentares generalistas, quatro são estritamente coprófagas e uma estritamente necrófaga. Foram verificadas correlações positivas entre a precipitação mensal e a abundância e a riqueza de espécies. O levantamento preliminar das espécies de Scarabaeinae da região de Brejo Novo contribuiu para aumentar o número de espécies registradas para o estado de Pernambuco e região Nordeste do Brasil.

PALAVRAS-CHAVE. Hábito alimentar; biodiversidade; ecologia; scarabaeinae.

Os escarabeíneos são popularmente conhecidos no Brasil como "rola-bostas", devido ao fato de alguns de seus representantes prepararem bola de excremento que é rolada até o local de consumo ou armazenamento, sendo essa uma característica peculiar do grupo (Halffter \& Matthews 1966; Halffter \& Favila 1993).

Os adultos e as larvas desta subfamília são detritívoros e utilizam principalmente fezes, carcaças e frutos em decomposição para alimentação, desempenhando assim uma importante função na dinâmica de nutrientes em diferentes tipos de ecossistemas (Hanski \& Cambefort 1991; Halffter \& Matthews 1966). A reprodução e a alimentação estão associadas, muitas vezes, ao transporte do recurso alimentar a um local distante da fonte original, evitando assim a competição com outros grupos de animais que utilizam os mesmos recursos, como por exemplo, dípteros e mamíferos, assim como outros escarabeíneos (Hanski \& Cambefort 1991).

Esses insetos formam uma comunidade bem definida em termos taxonômicos e funcionais, principalmente em florestas tropicais e savanas. Segundo Halffter \& Favila (1993) a comunidade destes besouros pode ser dividida em grupos de espécies denominados guildas. Os grupos podem ser estruturados de acordo com as estratégias de alocação de recursos alimentares (espécies paracoprídeas, telecoprídeas e 
endocoprídeas), o grau de generalização da dieta (coprófagas, necrófagas ou generalistas) e o padrão temporal de atividade (diurno, noturno, crepuscular e de atividade contínua). Essa divisão pode oferecer uma ferramenta bastante útil para avaliar modificações naturais ou antrópicas que uma comunidade sofre através de um determinado tempo, e desta forma esses insetos podem também ser utilizados como bioindicadores (Halffter et al. 1992; Davis et al.2001). Halffter \& Favila (1993) citaram algumas características que tornam essa subfamília importante para estudos que usam bioindicadores, dentre as quais estão a reciclagem dos excrementos e outros tipos de matéria orgânica, a abundância de seus representantes nos diferentes tipos de ecossistemas e a formação de guildas (funcional e taxonomicamente) que reagem à degradação de seu hábitat.

A especificidade de hábitat dos Scarabaeinae é outro fator importante quando se quer analisar a diversidade destes insetos em diferentes ambientes. Existem espécies que estão associadas a alguns fatores ambientais, como tipo de vegetação, microclima e solo. Com isso, a fragmentação das áreas de mata bem como a transformação destes ambientes em áreas de pastagem pode causar uma perda na diversidade de espécies e alterar drasticamente a estrutura da comunidade (Favila \& Halffter 1997).

Os escarabeíneos apresentam grande diversidade de espécies na região Neotropical, com cerca de 70 gêneros e 1.250 espécies conhecidas (Hanski \& Cambefort 1991). No Brasil foram registradas mais de 600 espécies incluídas em 49 gêneros, sendo 149 conhecidas no Nordeste brasileiro e apenas 12 registradas para o estado de Pernambuco (Vaz-deMello 2000).

Os brejos de altitude constituem disjunções da Floresta Tropical Perenifólia, dentro da Zona da Caatinga (AndradeLima 1960). Segundo Andrade-Lima (1960) estes ambientes podem ser considerados como "pequenas ilhas de florestas sobre maciços isolados", sendo a rigor "disjunções das matas empoleiradas nos altos das serras, apresentando taxas pluviométricas e higrométricas mais elevadas que nas áreas de caatinga onde se penetram, e menores que nas áreas do maciço florestal que as precede, submetidas a temperaturas com maior variação (maiores máximas e menores mínimas)". Estas áreas possuem hábitats originais úmidos, levando a uma diversificação da fauna e da flora (Andrade-Lima 1966, 1970).

O registro das espécies de escarabeíneos que ocorrem nas regiões de brejo de altitude do Nordeste brasileiro ainda é muito incipiente, por este motivo, o levantamento das espécies nestes ecossistemas, associado a estudos de estrutura de comunidade, contribuirão para ampliar o conhecimento da diversidade destes besouros.

O objetivo deste trabalho foi levantar preliminarmente a fauna de Scarabaeinae copro-necrófagos em uma região de brejo de altitude denominada Brejo Novo, localizada em Caruaru, Pernambuco, e analisar aspectos da estrutura da comunidade como sazonalidade, diversidade, equitabilidade, riqueza e abundância das espécies e tentar associar estas características às condições ambientais da região.

\section{MATERIALE MÉTODOS}

O estudo foi desenvolvido na Fazenda Encanto ( $8^{\circ} 42^{\prime} \mathrm{S}$, $35^{\circ} 15^{\prime} \mathrm{W}$ ), localizada na região de Brejo Novo, município de Caruaru, Pernambuco. A fazenda consiste em uma área com cerca de 120 ha, com vegetação constituída por uma floresta subperenifólia aparentemente bem conservada, intercalada por pequenas áreas de pastagens e circundada por vegetação xerófila de caatinga aparentemente bem conservada.

A região de Brejo Novo está localizada em uma área considerada remanescente de brejo de altitude e possui características biológicas e climáticas peculiares que a diferencia do tipo de ecossistema predominante da região (caatinga) (Tabarelli \& Santos 2004). Dentre estas características estão: clima subúmido, altitude aproximadamente de $660 \mathrm{~m}$, temperatura anual média $24^{\circ} \mathrm{C}$, precipitação anual em torno de $609 \mathrm{~mm}$, com período chuvoso correspondente aos meses de junho e julho e solos profundos argilosos, com alta capacidade de retenção de água.

Durante os meses de setembro de 2003 a julho de 2004 foram realizadas 10 coletas para captura dos insetos. $\mathrm{O}$ intervalo entre coletas foi de aproximadamente 30 dias, tendo cada coleta duração de 48 horas. Foram instaladas na área de pastagem junto à floresta subperenifólia 24 armadilhas de queda distanciadas $20 \mathrm{~m}$ e distribuídas ao redor da sede da propriedade. Nas armadilhas foram usados dois tipos de isca, 12 delas receberam aproximadamente $50 \mathrm{~g}$ de fezes humanas e as outras 12 a mesma quantidade de carne bovina em estado de putrefação. Essas iscas foram distribuídas alternadamente, não possuindo duas armadilhas seguidas o mesmo tipo de isca.

A armadilha consistiu em uma garrafa plástica cortada 20 $\mathrm{cm}$ acima da base possuindo $10 \mathrm{~cm}$ de diâmetro, sendo descartada a parte superior, no centro da abertura foi preso com arame um pequeno recipiente sem tampa servindo como

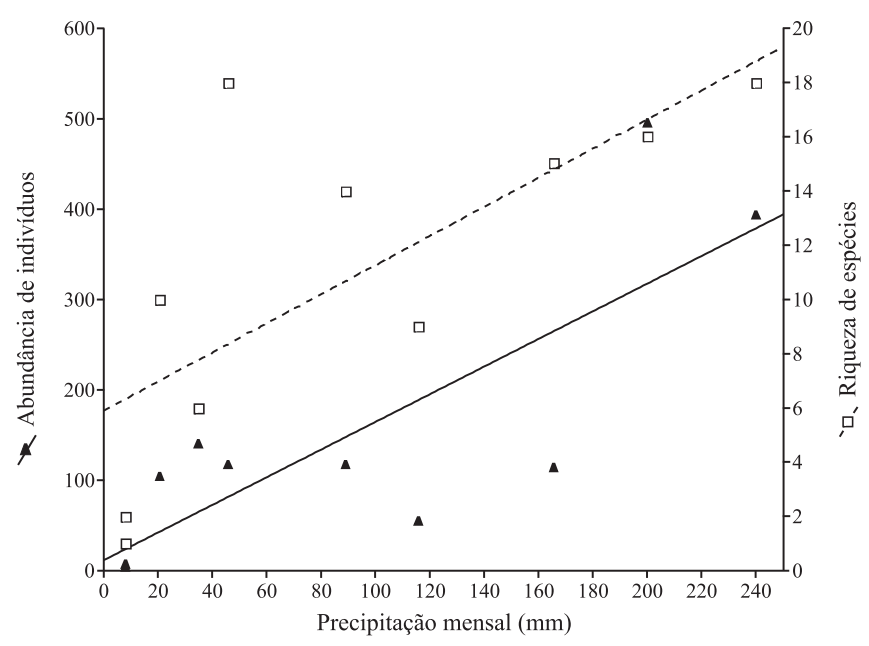

Fig. 1. Correlação entre a precipitação mensal, a abundância de indivíduos e a riqueza de espécies de Scarabaeinae (Coleoptera: Scarabaeidae) coletados entre setembro 2003 e julho 2004 na região de Brejo Novo, Caruaru, Pernambuco. 


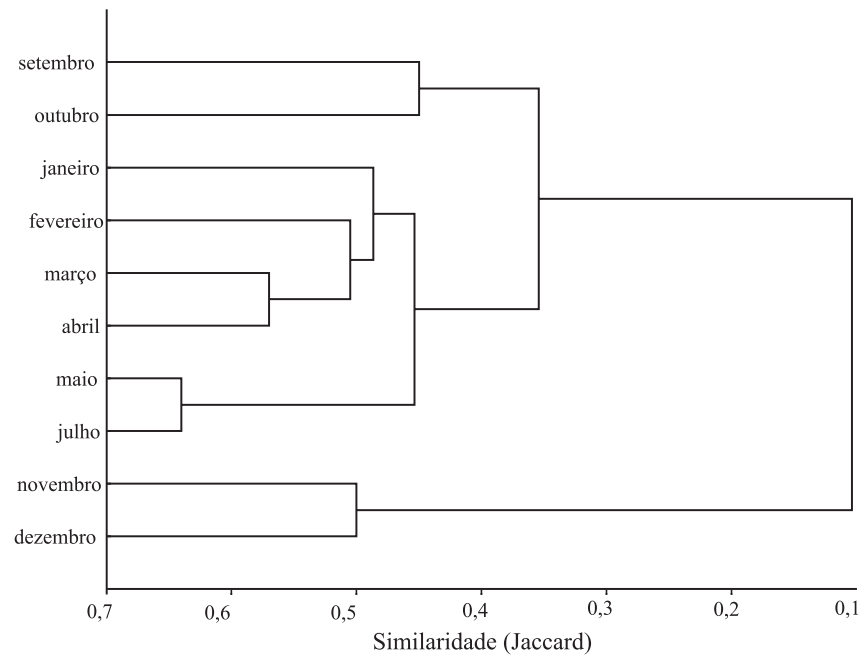

Fig. 2. Dendograma mostrando a similaridade entre os meses de coleta de Scarabaeinae (Coleoptera: Scarabaeidae) coletados entre setembro 2003 e julho 2004 na região de Brejo Novo, Caruaru, Pernambuco, baseado no Coeficiente de Jaccard.

porta iscas. Não foi colocado líquido no fundo das armadilhas devido à necessidade de obtenção dos insetos vivos para estudos paralelos de citogenética. As armadilhas foram enterradas com abertura ao nível do solo e sobre as mesmas foram colocados quadrados feitos com tela de $6 \mathrm{~cm}$ de diâmetro para impedir que animais de maior porte roubassem as iscas. A parte superior das telas foi forrada com plástico para proteção contra chuva.

O material coletado foi incorporado ao acervo do Departamento de Biologia, ICB/UPE, sendo sua identificação realizada através de comparação com espécimes da coleção "Adolph Hempel", Instituto Biológico (IBSP), e da coleção de Scarabaeidae do Laboratório de Entomologia (DSE/UFPB). Três espécies foram identificadas pelo pesquisador Fernando Z. Vaz-de-Mello.

Os dados de precipitação pluviométrica mensal para o período de estudo compreendido foram obtidos através do Laboratório de Meteorologia de Pernambuco (LAMEPE).

Para estabelecer o grau de abundância das espécies utilizou-se os critérios sugeridos por Colwell (1997), em que espécies com abundância de um a nove indivíduos são "raras", dentre estas, as que possuem apenas um único indivíduo são tidas como "singletons" e com dois indivíduos "doubletons". As espécies com abundância acima de dez indivíduos são ditas "comuns".

A análise da preferência alimentar foi feita através do programa Ecological Methodology (Kenney \& Krebs 2000), usando o índice estandardizado de Levins que se baseia na largura de nicho de cada espécie para os recursos alimentares estudados. O nível de significância adotado para todas as análises foi de 0,05 e apenas as espécies com mais de 20 indivíduos capturados foram analisadas para minimizar o viés. Para algumas espécies que apresentaram a quase totalidade dos seus indivíduos capturados em um determinado tipo de isca e tiveram abundância abaixo de 20 indivíduos foi usado o termo "possivelmente" antes da preferência alimentar.

Para análise da diversidade de espécies (índice de Shannon na base exponencial) e da equitabilidade (índice de Camargo) foi utilizado o programa Ecological Methodology v. 5. 2 (Kenney \& Krebs 2000). Foi realizado o teste não paramétrico de correlação de Spearman entre a precipitação mensal e os valores de abundância, riqueza, diversidade e equitabilidade de espécies, bem como o teste não paramétrico do quiquadrado, visando verificar se existia associação significativa entre o número de indivíduos capturados nas armadilhas iscadas com carne e fezes em função do período seco e chuvoso do ano (Zar 1996). Foi realizada uma análise de similaridade entre os meses de coleta a partir do coeficiente de similaridade de Jaccard, o qual inclui a presença e ausência das espécies em cada mês de coleta. Posteriormente estes dados foram incorporados em uma análise de aglomerados, realizada através do método de encadeamento médio. Para realização destes testes, organização dos dados e construção dos gráficos foi utilizado o programa Statistica for Windows v. 6 (Statsoft 1998).

\section{RESULTADOS EDISCUSSÃO}

Foi capturado um total de 1.540 indivíduos pertencentes a seis tribos, 12 gêneros e 28 espécies de Scarabaeinae. Destas espécies, 21 foram identificadas a nível específico e 14 são novos registros para o estado (Tabela I). As espécies registradas até 1998 eram: Canthon lituratus (Germar, 1824), Canthon staigi (Pereira, 1953), Coprophanaeus pertyi (d'Olsoufieff, 1924), Dichotomius crinicollis (Germar,1824), Dichotomius machadoi Martínez \& Pereira, 1967 (endêmica), Eurysternus caribaeus (Herbst, 1789), Malagoniella astyanax astyanax (Olivier, 1789), Ontherus aphodioides Burmeister, 1874, Ontherus appendiculatus (Mannerhein, 1829), Pseudocanthon xanthurum (Blanchard, 1843), Scybalocanthon cyanocephalum (Harold, 1868) (identificação incerta) e Sylvicanthon machadoi (Pereira \& Martínez, 1967) (endêmica) (Vaz-de-Mello, comunicação pessoal). O número de espécies registradas para o estado de Pernambuco foi ampliado de 12 (Vaz-de-Mello 2000) para 26 espécies atualmente. $\mathrm{O}$ escasso número de inventários deste grupo nos diferentes ecossistemas da região Nordeste do Brasil e estado de Pernambuco é o principal fator responsável pelo baixo número de espécies registradas.

Das seis tribos encontradas na região, Canthonini foi a melhor representada neste estudo com dez espécies, seguida pelas tribos Coprini (6), Ateuchini (5), Phanaeini (3), Onthophagini (3) e Eurysternini (1). O fato da tribo Canthonini estar bem representada, particularmente por espécies do gênero Canthon Hoffmannsegg, 1817, com a maioria de seus representantes copro-necrófagos, é reflexo de fatores históricos e ecológicos que incrementaram a diversidade de espécies desta tribo na região Neotropical (Vaz-de-Mello 1999).

O grande número de indivíduos registrados para a tribo Canthonini em relação às demais tribos, foi ocasionado pela 
Tabela I. Número de indivíduos por espécies e tribos de Scarabaeinae (Coleoptera: Scarabaeidae), coletados em armadilhas de queda iscadas com fezes humanas (F) e carne bovina apodrecida (C), de setembro de 2003 a julho de 2004, na região de Brejo Novo, Caruaru, Pernambuco. * = novos registros para Pernambuco.

\begin{tabular}{|c|c|c|c|}
\hline Tribos & \multicolumn{3}{|c|}{$\mathrm{N}^{\mathrm{o}}$ de Indivíduos } \\
\hline $\begin{array}{l}\text { Espécies } \\
\text { Ateuchini }\end{array}$ & $\mathrm{F}$ & $\mathrm{C}$ & Total \\
\hline Canthidium humerale (Germar, 1813) * & 13 & 1 & 14 \\
\hline Canthidium af. manni Arrow, 1913 & 22 & 0 & 22 \\
\hline Canthidium sp. 1 & 6 & 1 & 7 \\
\hline Canthidium sp. 2 & 2 & 0 & 2 \\
\hline Trichillum sp. & 9 & 1 & 10 \\
\hline Canthon lituratus (Germar, 1824) & 44 & 0 & 44 \\
\hline Canthon af. maldonadoi Martínez, 1951 & 1 & 1 & 2 \\
\hline Canthon mutabilis Harold, $1867 *$ & 12 & 19 & 31 \\
\hline Canthon af. carbonarius Harold, 1868 & 188 & 59 & 247 \\
\hline Canthon chalybaeus Blanchard, $1843 *$ & 164 & 426 & 590 \\
\hline Canthon sp. 1 & 1 & 0 & 1 \\
\hline Canthon sp. 2 & 0 & 1 & 1 \\
\hline Deltochilum irroratum (Laporte, 1840) * & 10 & 68 & 78 \\
\hline Deltochilum verruciferum Felsche, 1911 * & 3 & 2 & 5 \\
\hline $\begin{array}{l}\text { Malagoniella astyanax ssp. (Olivier, 1789) } \\
\text { Coprini }\end{array}$ & 3 & 1 & 4 \\
\hline Dichotomius bos (Blanchard, 1843)* & 9 & 0 & 9 \\
\hline Dichotomius nisus (Olivier, 1789) * & 143 & 11 & 154 \\
\hline Dichotomius semisquamosus (Curtis, 1845) * & 88 & 2 & 90 \\
\hline Dichotomius sericeus (Harold, 1867) * & 0 & 1 & 1 \\
\hline Ontherus digitatus Harold, $1868 *$ & 2 & 1 & 3 \\
\hline Ontherus sp. & 6 & 0 & 6 \\
\hline $\begin{array}{c}\text { Eurysternus hirtellus Dalman, } 1824 * \\
\text { Onthophagini }\end{array}$ & 29 & 30 & 59 \\
\hline Digitonthophagus gazella (Fabricius, 1787) * & 48 & 0 & 48 \\
\hline Onthophagus af. hirculus Mannerheim, 1829 & 6 & 1 & 7 \\
\hline $\begin{array}{r}\text { Onthophagus sp. } 1 \text { Phanaeini } \\
\end{array}$ & 3 & 0 & 3 \\
\hline Coprophanaeus jasius (Olivier, 1789) * & 0 & 27 & 27 \\
\hline Coprophanaeus pertyi (d'Olsoufieff, 1924) & 10 & 60 & 70 \\
\hline Diabroctis mimas (Linnaeus, 1758) * & 4 & 1 & 5 \\
\hline TOTAL & 826 & 714 & 1.540 \\
\hline
\end{tabular}

abundância da espécie Canthon chalybaeus Blanchard, 1843 $(\mathrm{N}=590)$. Esta espécie é dominante na época em que as condições climáticas da região são mais amenas, entretanto, nas coletas realizadas nos meses mais quentes e secos (novembro e dezembro) ela não foi capturada.

Os gêneros mais bem representados foram Canthon (7 espécies), Dichotomius Hope, 1838 (4) e Canthidium Erichson, 1847 (4). As espécies mais abundantes foram: Canthon chalybaeus (590) com 38,31\% do total de indivíduos coletados, Canthon af. carbonarius Harold, 1868 (247) com 16\%, Dichotomius nisus (Olivier, 1789) (154) com $10 \%$ e $D$. semisquamosus (Curtis, 1845) (90) com 5,84\%.

A espécie D. semisquamosus foi registrada em $100 \%$ das coletas, Canthon chalybaeus e Eurysternus hirtellus Dalman, 1824 em 80\%, Canthon af. carbonarius, D. nisus e
Digitonthophagus gazella (Fabricius, 1787) foram registradas em 60\%. Segundo Hanski \& Koskela (1978) a estabilidade de uma espécie é positivamente relacionada com a abundância e especialização ecológica. De acordo com isto, aparentemente estas são as espécies mais adaptadas ao ambiente estudado.

Analisando a abundância das espécies na região, a principal característica observada foi a presença de um grande número de espécies "raras" (15), sendo três destas "singletons", duas "doubletons" e dez com abundância entre três e 10 indivíduos. Foram registradas 13 espécies consideradas "comuns". A ocorrência de um grande número de espécies raras também foi verificada por Medri \& Lopes (2001) em um ambiente de floresta primária e área de pastagem adjacente. Este tipo de padrão foi também citado por Halffter (1991), como característico de florestas tropicais, onde poucas espécies são muito abundantes e muitas espécies são representadas por um número restrito de indivíduos.

Algumas espécies encontradas neste levantamento (Canthon lituratus, Canthidium af. manni Arrow, 1913, Deltochilum verruciferum Felsche, 1911, Dichotomius nisus, Malagoniella astyanax ssp. e Onthophagus af. hirculus Mannerheim, 1829) também foram coletadas em um ecossistema de Caatinga em duas localidades do Curimataú, Paraíba, durante o ano de $2003 \mathrm{com}$ armadilhas iscadas com carne de porco e fezes humanas (Hernández 2005). Também foram encontradas espécies registradas para o ecossistema de Mata Atlântica da Paraíba (Hernández 2003), as espécies comuns foram: Coprophanaeus jasius (Olivier, 1789), Dichotomius semisquamosus, D. sericeus Harold, 1867 e Eurysternus hirtellus Dalman, 1824.

O fato de algumas espécies coletadas neste levantamento serem encontradas em Caatinga e outras em remanescentes de Mata Atlântica pode ser explicado devido a região de Brejo Novo estar localizada em uma área de transição, ou ecótono, entre a Zona da Mata, composta por vegetação característica de Mata Atlântica, e o Sertão de Pernambuco, com predominância da Caatinga. Com isso, podemos observar neste local uma grande riqueza de espécies e diversidade de escarabeíneos associados à fauna do brejo de altitude.

Dentre os indivíduos capturados, 826 foram coletados em armadilhas iscadas com fezes humanas e 714 em carne bovina apodrecida (Tabela I). Através do teste do qui-quadrado foi observada uma diferença quanto ao número de insetos coletados por tipo de isca nos períodos seco e chuvoso $\left[\mathrm{X}^{2}=\right.$ 18,26; g. 1. $=1 ; \mathrm{p}<0,05]$. Para realização deste teste os dados

Tabela II. Número de indivíduos de Scarabaeinae (Coleoptera: Scarabaeidae), coletados na região de Brejo Novo, Caruaru, Pernambuco, de outubro de 2003 a julho de 2004, agrupados em período seco e chuvoso, para os dois tipos de iscas.

\begin{tabular}{lccc}
\hline \multirow{2}{*}{ Período } & \multicolumn{2}{c}{ Isca } & \multirow{2}{*}{ Total } \\
\cline { 2 - 3 } & Carne & Fezes & \\
\hline Seca & $376(37,6 \%)$ & $626(62,4 \%)$ & 1.002 \\
Chuva & $200(50,0 \%)$ & $200(50,0 \%)$ & 400 \\
Total & 576 & 826 & 1.402 \\
\hline
\end{tabular}


Tabela III. Número de indivíduos, riqueza de espécies, índices de diversidade (Shannon) e de equitabilidade (Camargo) dos Scarabaeinae (Coleoptera, Scarabaeidae) coletados na região de Brejo Novo, Caruaru, Pernambuco, de setembro de 2003 e julho de 2004.

\begin{tabular}{cccccccccccc}
\hline Mês/Ano & $\mathrm{IX} / 03$ & $\mathrm{X} / 03$ & $\mathrm{XI} / 03$ & $\mathrm{XII} / 03$ & $\mathrm{I} / 04$ & $\mathrm{II} / 04$ & $\mathrm{III} / 04$ & $\mathrm{IV} / 04$ & $\mathrm{~V} / 04$ & $\mathrm{VI} / 04$ & $\mathrm{VII} / 04$ \\
\hline Abundância & 139 & 103 & 6 & 4 & 393 & 495 & 116 & 113 & 116 & - & 55 \\
Riqueza & 6 & 10 & 2 & 1 & 18 & 16 & 18 & 15 & 14 & - & 9 \\
Shannon & 1,64 & 2,36 & 0,91 & - & 2,82 & 2,58 & 3,30 & 3,10 & 2,93 & - & 2,5 \\
Camargo & 0,40 & 0,39 & 0,83 & - & 0,29 & 0,30 & 0,41 & 0,44 & 0,41 & - & 0,48 \\
Precipitação mensal $(\mathrm{mm})$ & 35 & 21 & 8 & 8 & 240 & 200 & 46 & 166 & 89 & - & 116 \\
\hline
\end{tabular}

do mês de setembro de 2003 não foram computados devido à falta de informações, pois neste mês quase todas as iscas de carne foram roubadas por animais de grande porte. Foi considerada estação seca o período correspondente aos meses de setembro a fevereiro, e estação chuvosa o período entre março e agosto. No período chuvoso $50 \%$ dos insetos caíram nas armadilhas de fezes e $50 \%$ nas armadilhas de carne. Já na seca, 37,6\% foram atraídos para as armadilhas com carne e $62,4 \%$ para as armadilhas com fezes (Tabela II). Este padrão pode ser explicado devido à severidade da estação seca limitar a atratividade das iscas de carne, já que a carne pode ter sua decomposição alterada e exalar menos odor que as fezes (Louzada \& Lopes 1997). Segundo Escobar (1997), algumas espécies possuem preferência por certos tipos de excremento de acordo com a época do ano, o que pode estar relacionado a fatores reprodutivos nos Scarabaeinae.

A diversidade de espécies (índice de Shannon) capturadas foi menor nos meses de setembro, outubro e novembro (Tabela III). Para o mês de dezembro não puderam ser obtidos os valores de diversidade e equitabilidade devido a apenas uma espécie ter sido registrada na amostra. $O$ período em que a diversidade foi mais alta ocorreu entre os meses de janeiro a julho de 2004.

Através do teste de correlação de Spearman foi verificada uma correlação positiva significativa (Fig. 1) entre a precipitação mensal e a abundância de indivíduos $\left[\mathrm{r}_{S}=0,71 ; \mathrm{p}\right.$ $<0,05]$ ou seja, quando há um aumento na precipitação mensal a abundância aumenta. Este mesmo padrão foi observado para a riqueza $\left[\mathrm{r}_{S}=0,77 ; \mathrm{p}<0,05\right]$, havendo uma resposta positiva no número de espécies quando há um aumento da precipitação.

A vegetação de Brejo Novo sofreu uma marcada mudança no mês de janeiro devido a uma grande quantidade de chuvas após um período de estiagem e seca. Segundo Halffter \& Arellano (2001) mudanças ocorridas na vegetação podem interferir na oferta de alimento, que é um dos principais fatores conformadores da estrutura e diversidade de escarabeíneos. Por outro lado, a diversidade destes insetos responde não apenas ao tipo de alimento disponível, mas também às condições ambientais, como temperatura e umidade do ar, como observado em estudo realizado por Rodrigues \& Marchini (2000).

Os valores de equitabilidade (índice de Camargo) foram mais baixos nos meses de janeiro e fevereiro (Tabela III), isto se deve a ocorrência de uma grande quantidade de indivíduos pertencentes à espécie Canthon chalybaeus em detrimento das outras espécies nestes meses.

$\mathrm{Na}$ análise da preferência alimentar foi verificado um predomínio de espécies generalistas (7), seguido pelas espécies estritamente coprófagas (4) e necrófagas (1) (Tabela IV). Segundo Halffter \& Matthews (1966), a generalização da dieta é uma característica das comunidades de escarabeíneos de toda região Neotropical. Nossos resultados corroboram essa afirmação e são semelhantes aos encontrados por Louzada \&

Tabela IV. Preferência alimentar dos Scarabaeinae (Coleoptera, Scarabaeidae), da região de Brejo Novo, Caruaru, Pernambuco, de setembro de 2003 a julho de $2004{ }^{*}$ = espécies com abundância menor que 20.

\begin{tabular}{|c|c|c|}
\hline Tribo & Espécies & Preferência Alimentar \\
\hline \multirow[t]{4}{*}{ Ateuchini } & Canthidium humerale (Germar, 1813)* & Possivelmente coprófago \\
\hline & Canthidium af. manni Arrow, 1913 & Coprófago \\
\hline & Canthidium sp. $1 *$ & Possivelmente coprófago \\
\hline & Trichillum sp. * & Possivelmente coprófago \\
\hline \multirow[t]{5}{*}{ Canthonini } & Canthon lituratus (Germar, 1824) & Coprófago \\
\hline & Canthon mutabilis Harold, 1867 & Generalista \\
\hline & Canthon af. carbonarius Harold, 1868 & Generalista (preferencialmente coprófago) \\
\hline & Canthon chalybaeus Blanchard, 1843 & Generalista \\
\hline & Deltochilum irroratum (Castelnau, 1840) & Generalista (preferencialmente necrófago) \\
\hline \multirow[t]{3}{*}{ Coprini } & Dichotomius bos (Blanchard, 1843) * & Possivelmente coprófago \\
\hline & Dichotomius nisus (Olivier, 1789) & Generalista (preferencialmente coprófago) \\
\hline & Dichotomius semisquamosus (Curtis, 1845) & Coprófago \\
\hline Eurysternini & Eurysternus hirtellus Dalman, 1824 & Generalista \\
\hline \multirow[t]{2}{*}{ Onthophagini } & Digitonthophagus gazella (Fabricius, 1787) & Coprófago \\
\hline & Onthophagus af. hirculus Mannerheim, $1829 *$ & Possivelmente coprófago \\
\hline \multirow[t]{2}{*}{ Phanaeini } & Coprophanaeus jasius (Olivier, 1789) & Necrófago \\
\hline & Coprophanaeus pertyi (d'Olsoufieff, 1924) & Generalista (preferencialmente necrófago) \\
\hline
\end{tabular}


Lopes (1997) na comunidade de Viçosa, Minas Gerais, e aos de Morón et al. (1985) em Chiapas, México. Apesar disso, consideramos que este tipo de estruturação pode ser drasticamente alterado por ações antrópicas ou naturais que uma determinada região venha apresentar, como observado por Halffter et al. (1992) em uma área onde o desmatamento e a transformação da paisagem original geraram um mosaico de áreas de pastagem e alterou o tipo de espécies predominantes, favorecendo o aparecimento e a dominância de espécies coprófagas.

A análise de similaridade entre os meses de coleta mostra que os meses mais diferentes de todos os outros, formando um grupo afastado, foram novembro e dezembro, por apresentar um número muito baixo de espécies (Fig. 2). Também vemos um segundo grupo constituído pelos meses de setembro e outubro, com uma riqueza de espécies média. E um terceiro grupo corresponde ao período de janeiro a julho, no qual a quantidade de espécies foi mais alta (entre 9 e 18 espécies). Este último grupo está subdividido em dois subgrupos, em que os meses de maio e julho aparecem juntos apresentando menos de 15 espécies, com muitas espécies em comum, enquanto nos meses do outro subgrupo foram registradas entre 15 e 18 espécies. O grupo que apresentou a maior riqueza de espécies coincidiu com as maiores precipitações no período do estudo. Isso corrobora a afirmação de que a atividade das espécies de Scarabaeinae da comunidade local é maior nos meses mais úmidos, reforçando a hipótese de que alterações ambientais podem ter grande influência na atividade das espécies e na estruturação da comunidade de diferentes regiões.

Agradecimentos. Ao Conselho Nacional de Desenvolvimento Científico e Tecnológico - CNPq e a Fundação de Amparo à Ciência e Tecnologia do Estado de Pernambuco - FACEPE (Processo ${ }^{\circ}$ 006/2003: PPP / MCT / CNPq / CT-INFRA / FACEPE, de R.C Moura); ao CNPq/ PIBIC/UPE (bolsa de Iniciação Científica, de F.A.B. Silva); A CAPES pela bolsa de M.I.M. Hernández através do Programa de Apoio a Projetos Institucionais com a participação de Recém Doutores (PRODOC); a Fernando Z. Vaz-de-Mello, pela identificação de algumas espécies.

\section{REFERÊNCIAS}

Andrade-Lima, D. 1960. Estudos fitogeográficos de Pernambuco. Arquivos do Instituto de Pesquisas Agronômicas 5: 305-341.

Andrade-Lima, D. 1966. Esboço fitoecológico de alguns brejos de Pernambuco. Boletim Técnico do Instituto de Pesquisas Agronômicas de Pernambuco 8: 1-27.

Andrade-Lima, D. 1970. Recursos Vegetais de Pernambuco. Boletim Técnico do Instituto de Pesquisas Agronômicas de Pernambuco 41: 1-32.

Colwell, R. K. 1997. EstimateS: Statistical estimation of species richness and shared species from samples. Version 5. Disponível em <http://viceroy.eeb.uconn.edu/ estimate>.

Davis, A .J.; J. D. Holloway; H. Huijbregts; J. Krikken; A. H. KirkSpriggs \& S. L. Sutton. 2001. Dung beetles as indicators of change in the forests of northern Borneo. Journal of Applied Ecology 38: $593-616$.

Escobar, F. 1997. Estudio de la comunidad de coleópteros coprófagos

Recebido em 21/09/2006; aceito em 22/03/2007
(Scarabaeidae) en un remanente de bosque seco al norte del Tolima. Caldasia 19: 419-430.

Favila, M. E. \& G. Halffter. 1997. The use of indicator groups for measuring biodiversity as related to community structure and function. Acta Zoológica Mexicana 72: 1-25.

Halffter, G. 1991. Historical and ecological factors determining the geographical distribution of beetles (Coleoptera: Scarabaeidae: Scarabaeinae). Folia Entomológica Mexicana 82: 195-238.

Halffter, G. \& M. E. Favila. 1993. The Scarabaeinae (Insecta: Coleoptera) an animal group for analyzing, inventorying and monitoring biodiversity in tropical rainforest and modified landscapes. Biology International 27: 15-21.

Halffter, G.; M. E. Favila \& V. Halffter. 1992. A comparative study of the structure of the scarab guild in Mexican tropical rain forests and derived ecosystems. Folia Entomológica Mexicana 84: 131-156.

Halffter, G. \& L. Arellano. 2001. Variación de la diversidad en especies de Scarabaeinae (Coleoptera: Scarabaeidae) como respuesta a la antropización de un paisaje tropical, p. 35-53. In: J. L. NavarreteHeredia, H. E. Fierros-López \& A. Burgos-Solorio (eds.). Tópicos sobre Coleoptera de México. Guadalajara, Universidad de Guadalajara, $108 \mathrm{p}$.

Halffter, G. \& E .G. Matthews. 1966. The Natural History of Dung Beetles of the Subfamily Scarabaeinae (Coleoptera, Scarabaeidae). Folia Entomológica Mexicana 12-14: 1-312.

Hanski, I. \& Y. Cambefort. 1991. Dung Beetles Ecology. Princeton, New Jersey, $481 \mathrm{p}$.

Hanski, I. \& H. Koskela. 1978. Stability, abundance, and niche width in the beetle community inhabiting cow dung. Oikos 31: 290-298.

Hernández, M. I. M. 2003. Riqueza de Besouros Escarabeídeos em duas áreas de Floresta Atlântica no Estado da Paraíba. In: Anais de trabalhos completos do VI Congresso de Ecologia do Brasil Simpósio \& Floresta Pluvial Tropical Atlântica, Fortaleza, p. 300-302.

Hernández, M. I. M. 2005. Artrópodes: Besouros Scarabaeidae (Coleoptera) do Curimataú, Paraíba, Brasil, p. 369-380. In: F. S. Araújo, M. J. N. Rodal \& M. R. V. Barbosa (eds.). Análise das Variações da Biodiversidade do Bioma Caatinga para Suporte a Estratégias Regionais de Conservação. Brasília, PROBIO, Ministério do Meio Ambiente, 434 p.

Kenney, A. J. \& C. J. Krebs. 2000. Programs for Ecological Methodology, 2nd ed. Vancouver, Dept. of Zoology, University of British Columbia.

Louzada, J. N. C. \& F. S. A. Lopes. 1997. A comunidade de Scarabaeidae copro-necrófagos (Coleoptera) de um fragmento de Mata Atlântica. Revista Brasileira de Entomologia. 41: 117-121.

Medri, I. M. \& J. Lopes. 2001. Scarabaeidae (Coleoptera) do parque estadual Mata dos Godoy e de áreas de pastagens, no norte do Paraná, Brasil. Revista Brasileira de Zoologia 18: 135-141.

Morón, M. A.; F. J. Villalobos \& C. Deloya. 1985. Fauna de coleópteros Lamelicornios de Boca de Chajul, Chiapas, México. Folia Entomológica Mexicana 66: $57-118$.

Rodrigues, S. R. \& L. C. Marchini. 2000. Ocorrência de besouros coprófagos em dois diferentes ambientes. Revista Brasileira de Entomologia 44: 35-38.

Statsoft. 1998. Statistica for Windows. Tulsa, StatSoft

Tabarelli, M. \& A. M. M. Santos. 2004. Uma breve descrição sobre a história natural dos Brejos Nordestinos, p. 17-24. In: K. C. Porto; J. J. P. Cabral \& M. Tabarelli (eds.). Brejos de Altitude em Pernambuco e Paraíba. Brasília, Ministério do Meio Ambiente, $324 \mathrm{p}$.

Vaz-de-Mello, F. Z. 1999. Scarabaeidae s. str. (Coleoptera: Scarabaeoidea) de um fragmento de floresta amazônica no estado do Acre, Brasil: Taxocenose. Anais da Sociedade Entomológica do Brasil 28: 447-453.

Vaz-de-Mello, F. Z. 2000. Estado atual de conhecimento dos Scarabaeidae s. str. (Coleoptera: Scarabaeoidea) do Brasil, p. 183195. In: F. Martin-Piera; J. J. Morrone \& A. Melic (eds.). Hacia un Proyecto CYTED para el inventario y estimación de la diversidad Entomológica en Iberoamérica: PrIBES-2000, Zaragoza, Sociedad Entomológica Aragonesa \& CYTED, m3m: Monografias Tercer Milenio, v. 1, 326 p.

Zar, J. 1996. Biostatistical Analysis. Upper Saddle River, PrenticeHall, $662 \mathrm{p}$. 